Corresponding Author:

Jonathan Farley

Ricardo Plc

Southam Road

Radford

Leamington Spa

CV31 1FQ

07787872886

Jonathan.farley@ricardo.com 


\title{
Performance evaluation of multilayer thin film coatings under mixed rolling-sliding dry contact conditions
}

\author{
Jonathan Farley ${ }^{1,2}$ Luiz C. Wrobel ${ }^{2}$, Ken $\mathrm{Mao}^{3}$ \\ ${ }^{1}$ Currently at Ricardo Plc, Southam Road, Radford, Leamington Spa, CV31 1FQ \\ ${ }^{2}$ School of Engineering \& Design, Brunel University, Uxbridge, UB8 3PH \\ ${ }^{3}$ Department of Mechanical Engineering, University of Warwick, Coventry, CV4 7AL
}

\begin{abstract}
This paper focuses on the application of advanced finite element analysis to simulate the response of complex multilayer coatings when subjected to mixed rolling-sliding dry contact conditions, typical of those experienced by heavily loaded gear tooth flanks. In this study, a versatile model is developed to investigate the response of a variety of complex coatings. Through the investigation of three advanced surface coatings the developed model is shown to offer detailed subsurface stress and strain information, quantifying the benefits provided by the application of each coating.
\end{abstract}

A number of rolling contact fatigue tests was also conducted to complement the computational simulations. An adapted twin disc testing machine was used to investigate the progressive wear rates of the three advanced surface coatings when subjected to mixed rolling-sliding contact at high load and in dry conditions. The result is a clear indication that advanced surface coating techniques can provide significant improvements in wear resistance and surface durability. From the rolling contact fatigue experiments conducted in this study, a multilayer Tungsten Carbide/Carbon coating was shown to provide the most significant increase in surface durability with a 16 -fold reduction in measured weight loss compared to the uncoated substrate over the 145,000 cycle test duration investigated.

\section{KEY WORDS}

Coating simulation, contact mechanics, DLC, Abaqus, FEA, gears, motorsport 


\section{INTRODUCTION}

The automobile industry is one of the largest sectors of the global economy. In 2005, the global automobile manufacturing industry was valued at over $\$ 1.61$ trillion while the global automotive parts and equipment sector was valued at $\$ 522.8$ billion [1]. In 2003, an estimated 41 million new passenger vehicles rolled off the world's assembly lines, 16.6 million of which were sold in the USA alone [2]. Today, an estimated 10 million new cars enter the roads of China each year, a five fold increase since 2002. This significant increase in vehicle use has put additional demands on crude oil no doubt contributing to its recent market increase in price, some $40 \%$ from $2005-2008$. As a result, many automotive manufactures are making significant investments in various technologies offering improvements in fuel economy. One of these emerging markets is the development and application of advanced surface coating techniques.

A recent study by Taylor [3] suggested that as little as $25 \%$ of the available power from the combustion of fuel is directly converted to driving power to the wheels. An estimated $60 \%$ is lost through thermal exchanges and $15 \%$ to mechanical losses. Of these mechanical losses, $45 \%$ is attributed to the friction in the piston assembly, 20\% to pumping losses, $25 \%$ to bearings and a final $10 \%$ to the valve train [3]. Advanced surface coatings have been used in many industries for the past decade with great success. Offering reduced surface friction and improved wear resistance for nominal weight gain, surface coatings could be a rapid solution to significantly improving fuel economy. A reduction of just $10 \%$ in the mechanical losses alone could result in an improvement of $1.5 \%$ in total fuel consumption. Based on an average 35 mile per gallon fuel consumption over a typical 180,000 mile life cycle, this would yield savings in the order of 400 litres, over $£ 500$ at current UK fuel prices.

Determining the performance improvements provided by the application of complex coatings can be a difficult task. As a result, much of the coating development process is essentially by 'trial and error', requiring extensive and costly physical experimentation. With many advanced coatings containing numerous constituent layers, each with different mechanical properties and thicknesses, improving the 
performance of a coating may result in a multitude of combinations, the effect of each becoming extremely difficult to quantify.

This paper focuses on the application of advanced finite element analysis to simulate the response of complex multilayer coatings when subjected to mixed rolling-sliding contact conditions, typical of those experienced by gear tooth flanks. The result is a highly versatile simulation method capable of modelling a variety of complex coatings whilst providing rapid modification of each layer's mechanical properties and contact conditions. In this paper, the developed model is used to simulate and compare the response of three advanced surface coatings currently in use for specific motorsport applications. Through this study, the model can be seen to offer a direct comparison of each coating, identifying the potential improvements provided by each and highlighting the coatings' effect on the contact loads and material subsurface stress field.

The second part of this paper focuses on a detailed wear rate comparison conducted by using disc samples coated with the aforementioned coatings. Although extensive research has been conducted into gear flank failure mechanism, very little experimental data are available in the literature related to the wear characteristics and failure modes of coatings under complex rolling-sliding contact conditions. To help quantify the potential benefits achieved through the applications of coatings, a number of experimental investigations were conducted under mixed rolling-sliding contact conditions comparing the wear rates and surface failure of the same three advanced surface coatings. A twin-disc testing rig was adopted to induce mixed rolling-sliding wear on a selection of test samples, providing a direct comparison of each coating under the same testing conditions.

\section{MULTILAYER CONTACT MODELLING}

The subject of contact mechanics and the modelling of contact between two surfaces have become a significant part of engineering design, a trend set to continue with the development of ever more advanced surface modification techniques. However, the use of Finite Element (FE) packages to model contact problems is still limited in industry due to both software limitations and the frequent 
problems associated with model stability and solution convergence. With the advances made in FE codes and through the correct application of a number of simulation aids, accurate modelling of realistic contact problems has become more viable. Even so, investigations into the response of coated surfaces remain relatively sparse. Komvopoulos pioneered the application of FE to layered contact problems through an investigation of the improvements obtained through surface nitriding [4]. Since then, significant progress has been made with many studies conducted into the effects of surface roughness [5], varied mechanical properties [6] and complex multilayer coatings [7]. Yet, significant gaps still exist in the knowledge of how advanced multilayer coatings respond to complex surface loading conditions. In an attempt to improve that knowledge, and in particular, to investigate the comparable effect of a number of different coatings, an advanced FE software (Abaqus) was applied to investigate the response of three advanced surface coatings currently being utilised in a number of applications for motorsport engine and driveline components $[8,9]$.

\section{Modelling mixed rolling-sliding contact problems}

Abaqus is an advanced general purpose FE package. Initially developed to model the response of solids and structures to loads, Abaqus includes a very extensive material and element library making it capable of simulating a number of highly complex problems. In addition to a very robust $\mathrm{FE}$ code are a number of specialist capabilities such as the ability to simulate thermal loading, electrical conductivity, gaskets and seals, impact and vibration, all within the realms of linear and non-linear analysis. Due to its non-linear capabilities, Abaqus is particularly suited for modelling complex contact problems, such as the highly non-linear contact experienced by the teeth of gears transmitting load.

Consider the contact of two involute spur gears (figure 1). As the driven gear begins to mesh with the driving gear, contact will first occur between the root of the driver and the tip of the driven gear. Due to the profile of the teeth, high slipping contact occurs as the teeth flanks slide past each other during rotation. At the pitch point, the contact becomes pure rolling and the teeth pivots around their common centres, creating zero relative motion. Lastly, as the teeth disengage, high sliding contact occurs once more as the driving gears tip contacts the driven gears root. This mixed rolling-sliding 
contact is common in many applications and must therefore be an important consideration when developing real world simulations.

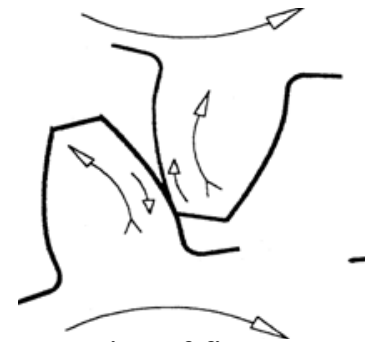

Point of first contact

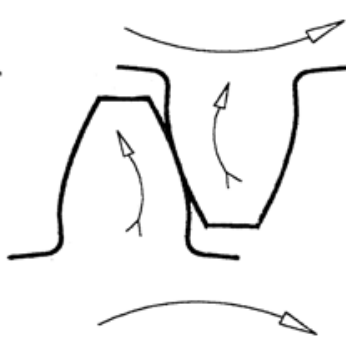

Pitch point

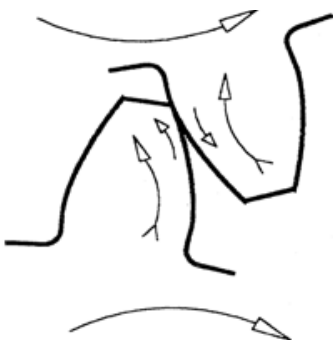

Point of last contact

\section{$\neg$ Direction of roll \\ $\rightarrow \quad$ Direction of friction forces}

Figure 1 - Mechanics of gear tooth contact [10]

Abaqus is particularly suited to modelling complex contact problems through its utilisation of a 'proximity tolerance' method. Contact surfaces are defined within the model and designated as either 'master' or 'slave'. Both the master and slave can include a number of surfaces from within the model but must be selected according to the following rules: the master surface must be chosen as the surface with the highest material stiffness and the master surface must have the coarsest mesh density [11]. Following the correct definition of the contact pair, surface contact attributes, such as interfacial friction, can be assigned to the model to appropriately replicate the desired contact problem. A proximity tolerance must also be defined for each contact pair. When nodes from the contact pair are found to be within this tolerance, contact is assumed and loads are transmitted between the surfaces. However, when the nodes are found to be outside the contact tolerance, no forces or interactions are applied. This contact calculation is performed for each contact pair at every iteration of every time step, resulting in the potential to accurately model highly non-linear surface contact. Such extensive proximity checks are highly demanding on computational resources making the choice of step size, mesh density and element selection critical. 


\section{Multilayer Contact Model}

The model developed in this study was created to investigate the response of multi-layer thin film coatings to mixed rolling-sliding contact. Each of the coatings investigated is extremely thin, with coating depths of no more than $3 \mu \mathrm{m}$. To accurately simulate the response of such a thin coating requires the use of extremely high mesh densities. For this reason, the model is simulated with twodimensional planar shell elements. Each coating layer is modelled as a separate component and is joined to the substrate using a constraint termed 'tying' (figure 2). This joins adjacent surfaces, removing any relative motion between them, giving an effective join of two dissimilar surfaces [11]. This function gives the user the ability to simulate a number of layered media with differing mechanical properties in contact, each with independent mesh densities and element selections. This is ideally suited for modelling coated surfaces and allows accurate mesh refinement at the areas of interest keeping the simulation as efficient as possible. Modelling each coating layer as a separate component also allows rapid modification of the mechanical properties of each layer.

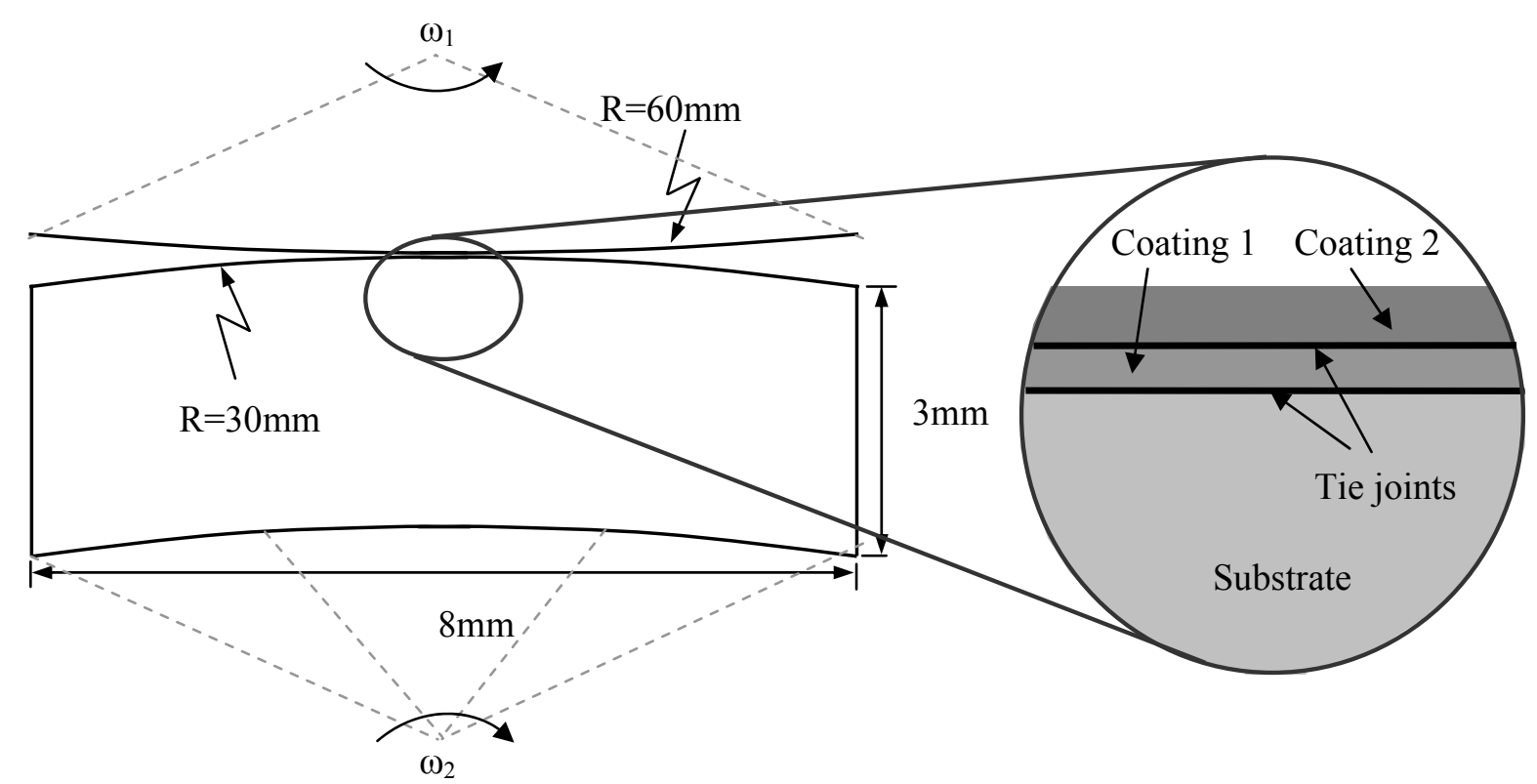

Fig 2 - Schematic of rolling-sliding coating contact model

Obtaining stable solutions for non-linear contact models in Abaqus can be challenging. Abaqus applies the designated loads to the model in progressive increments. At the beginning of each step, a 
small increment of the final load is applied and the contact area for each contact pair is determined. Where contact does exist, forces are transferred at the interfaces and the model solved. Once converged, a larger increment of the load is applied and the same calculations made. This continues progressively until the target maximum load has been reached. For complex contact models, choosing the correct increment is critical as too large an increment will result in solution cut backs, automatic increment reductions by the Abaqus solver to try and determine a stable solution. Conversely, too small an increment will result in an unnecessarily slow and computationally extensive solution. One method that was successfully used in this model to improve solution stability is that of applying the boundary conditions and loads in a number of discrete steps (figure 3). In the first step, all of the boundary conditions are applied to the model, fixing the movement of the indenter and sample in all directions. Next, a small vertical contact load is applied to the model through the indenter so an initial contact patch can be defined. Once stable, a larger vertical load is applied to generate the target contact pressure. Lastly, slip is introduced into the contact by applying a different angle of rotation to both the indenter and sample.

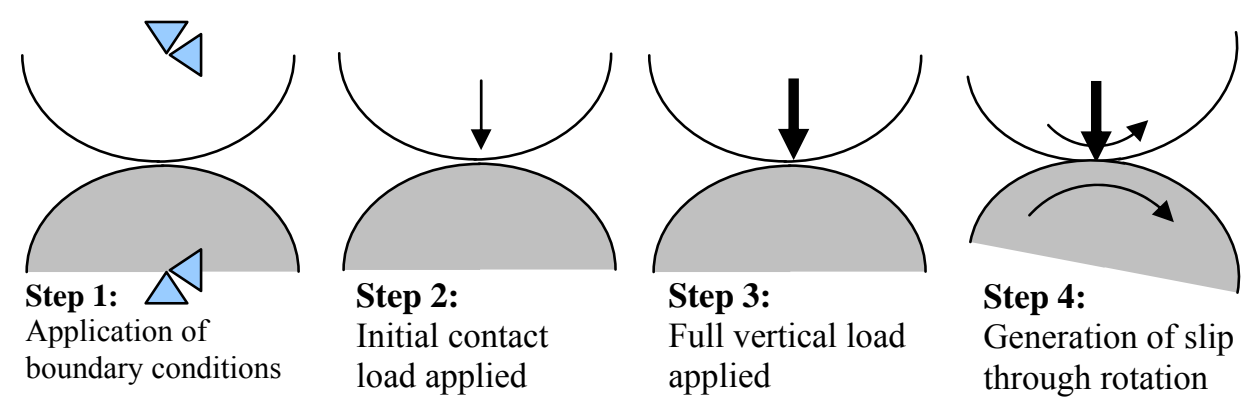

Figure 3 - Multi-step rolling-sliding contact analysis procedure

The primary purpose of the model developed in this study is to provide an accurate yet versatile simulation technique generating rapid solutions that can be used to improve the understanding of multilayer coatings response to specific loads. To keep the simulation times as short as possible, the model was limited to two dimensions. Two-dimensional linear plane strain elements (CPE4R) were used throughout the model with an increased mesh density focused at the interface between the 
substrate and coating layer. Each coating layer was simulated as elastic perfectly-plastic with the Coulomb friction penalty method used to model the surface interaction.

\section{CONTACT MODEL APPLICATION}

The multilayer contact model has been used to conduct a comparison study into the beneficial effects provided through the application of three commercially available surface coatings currently finding use in a number of motorsport applications. The first coating, Coating A, is a single layer TitaniumNitride coating, commonly used in tooling applications, offering excellent resistance to wear from its extremely hard TiN composition. The second coating, Coating B, is a multilayer Diamond-Like Carbon coating that utilises both Chromium and Chromium-Nitride interlayers. With high resistance to wear and low interfacial friction properties, this coating has been shown to perform well in highly loaded, high sliding velocity applications such as cam shafts and valve tappet systems. Lastly, Coating $\mathrm{C}$ is a multilayer Tungsten Carbide/Carbon coating that utilises a very thin Chromium interlayer to improve adhesion to the substrate. Coating $\mathrm{C}$ has been found to perform well in mixed rolling-sliding applications with its high carbon content offering reduced friction and some degree of self-lubrication. Each coating has been simulated as if applied to a high quality through hardened high speed steel. Table 1 contains a detailed breakdown of each of the coatings, highlighting the various mechanical properties used for each layer. To offer a direct comparison of the coated systems and allow the identification of improvements achieved by the addition of surface layers, each of the model simulations was conducted under the same boundary and loading conditions within Abaqus using full non-linearity modelling, and with elastic-perfectly plastic materials constraints. To simulate the high contact stresses experienced by gear teeth flanks, each of the coatings was loaded to a maximum Hertzian contact pressure $\left(\mathrm{P}_{0}\right)$ of $1 \mathrm{GPa}$, and a slide-roll ratio of $10 \%$ used. 
Table 1 - Multilayer coating mechanical properties

\begin{tabular}{|c|c|c|c|c|c|c|}
\hline Simulation & $\begin{array}{c}\text { Coating } \\
\text { layer }\end{array}$ & $\begin{array}{c}\text { Thickness } \\
\mathrm{t}(\mu \mathrm{m})\end{array}$ & $\begin{array}{l}\text { Young's } \\
\text { Modulus } \\
\text { E (GPa) }\end{array}$ & $\begin{array}{c}\text { Poisson's } \\
\text { Ratio }\end{array}$ & $\begin{array}{c}\text { Hardness } \\
\text { (HV) }\end{array}$ & $\begin{array}{c}\text { Friction } \\
\qquad \mu\end{array}$ \\
\hline $\begin{array}{c}\text { Uncoated } \\
\text { (i.e. substrate) }\end{array}$ & - & - & 206 & 0.29 & 748 & $0.7 *$ \\
\hline Coating A & TiN & 3.00 & 480 & 0.25 & 2300 & $0.3^{*}$ \\
\hline \multirow{3}{*}{ Coating B } & DLC & 1.88 & 170 & 0.20 & 2300 & \multirow{3}{*}{$0.1^{*}$} \\
\hline & $\mathrm{CrN}$ & 2.69 & 210 & 0.20 & 1750 & \\
\hline & $\mathrm{Cr}$ & 0.30 & 140 & 0.22 & 800 & \\
\hline \multirow{2}{*}{ Coating C } & $\mathrm{WC} / \mathrm{C}$ & 1.00 & 250 & 0.25 & 1050 & \multirow{2}{*}{$0.1^{*}$} \\
\hline & $\mathrm{Cr}$ & 0.20 & 140 & 0.22 & 800 & \\
\hline
\end{tabular}

\section{CONTACT MODEL RESULTS}

Typical FE meshes used in the simulations are shown in figure 4, while figure 5 shows Von Mises stress contour plots produced for each of the models. The beneficial effect of the coatings on the subsurface stress field is marked. With its high coefficient of friction under dry conditions, the uncoated model shows the peak stresses occurring directly below the point of contact at the surface of the substrate. Stress concentrations like these directly below the contact will have a detrimental effect on the durability of the component surface, causing rapid surface damage and fatigue failure through pitting and spalling. With Coating A applied, the hard TiN coating has the effect of containing the high contact stresses within the coating layer, reducing the stress in the softer substrate material. Due to its increased hardness, the TiN layer is seen to act as a barrier, reducing the subsurface stresses experienced by the substrate. The application of both Coating B and Coating $\mathrm{C}$ is shown to have a different effect. Due to their high carbon content, both coatings offer significantly reduced friction coefficients. This aids in both reducing the contact stresses and drawing the stress concentration away from the surface and into the material subsurface region, greatly reducing the potential for surface damage. Coating $\mathrm{C}$, with its softer $\mathrm{WC} / \mathrm{C}$ layer, can be seen to offer the best overall stress reduction, avoiding the peak stress concentration present in the significantly harder DLC layer used in Coating B. 


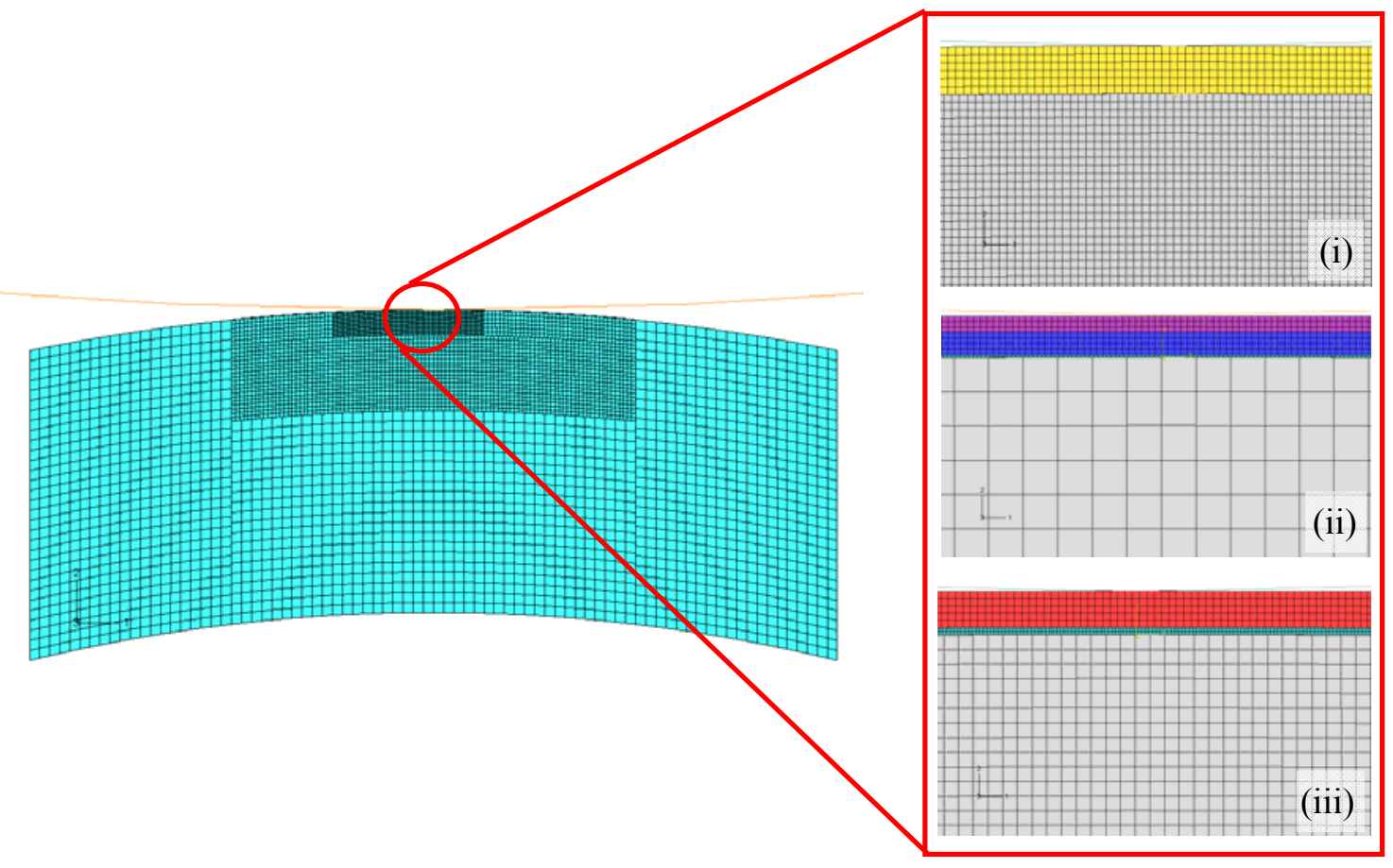

Figure 4 - Multilayer contact FE meshes: (i) Coating A; (ii) Coating B; (iii) Coating C
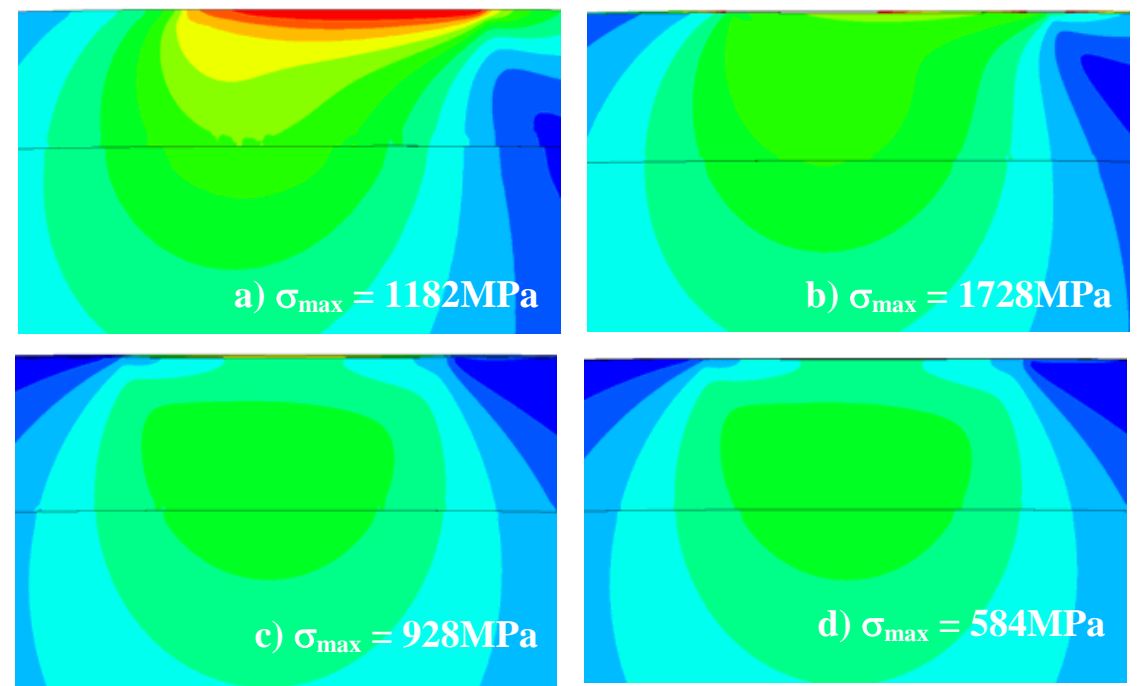

Figure 5 -Von Mises stress contour plots; a) Uncoated, b) Coating A [TiN], c) Coating B [DLC] d) Coating C [WC/C] 
Figure 6 shows a plot of the stress variation (normalised by $\mathrm{P}_{0}$ ) with depth taken at the contact centre for each of the model simulations. This figure provides a direct comparison on how the subsurface stresses change with depth from the contact surface and clearly demonstrates that, although Coating A shows an overall increase in contact stress directly at the surface, the peak stresses are contained within the first $2 \mu \mathrm{m}$ of the TiN coating, after which the stresses tail off towards the interface with the substrate. When compared to the stresses recorded at the same depth in the uncoated model, the substrate stresses for the TiN model were almost halved. For Coating B, the subsurface stress increases slightly in the harder $\mathrm{CrN}$ second layer before tapering off rapidly in the third Chromium layer as the depth nears the substrate interface. Lastly, Coating $C$ shows that very low stresses occur at the contact surface with a minimal change between the stress in the coating and substrate. This smooth transition will serve to aid adhesion between the coating and substrate, reducing the potential for coating delamination. Figure 5 shows that, for both Coating B and Coating C, the maximum stress occurs well below the contact surface, at a depth of around $30-40 \mu \mathrm{m}$ (see figures $5 \mathrm{c}$ and $5 \mathrm{~d}$ ). This will have a profound effect on the surface durability of the coating, with the surfaces stresses remaining low and the higher stresses contained within the substrates subsurface region.

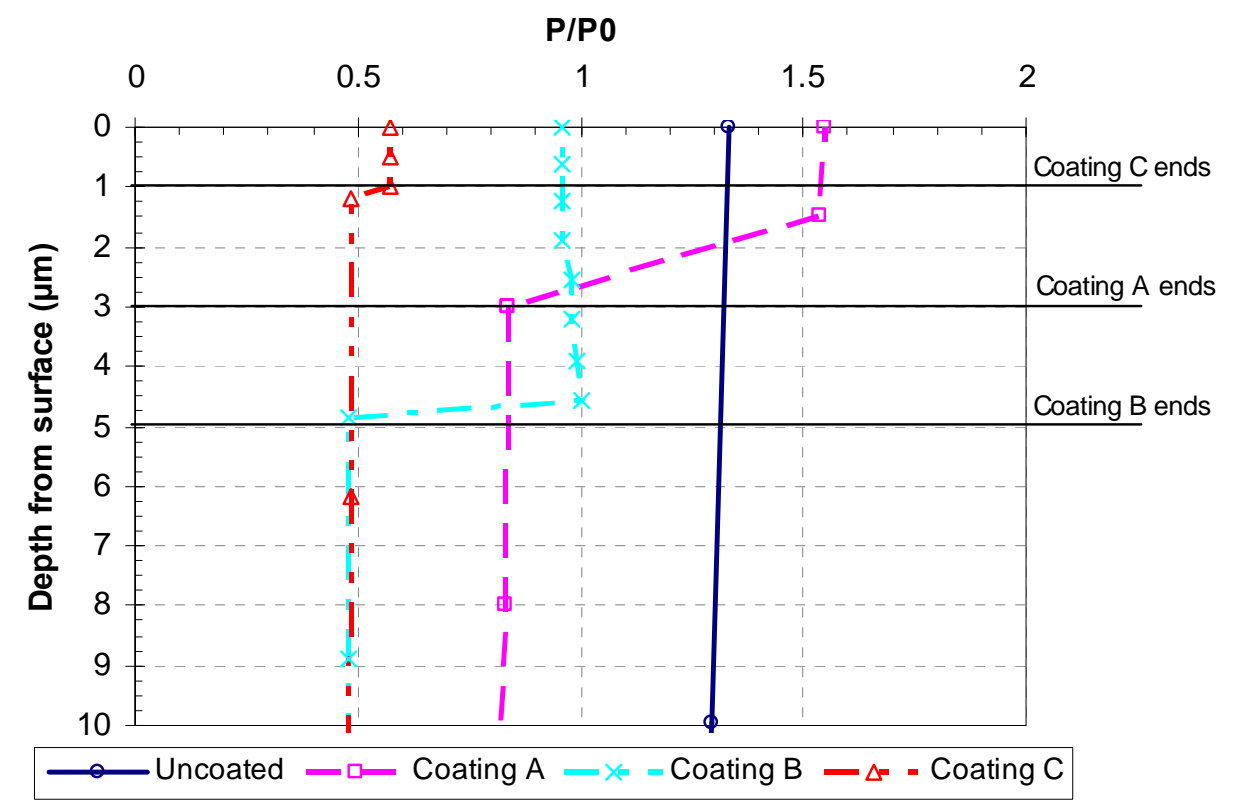

Figure 6 - Stress variation with depth for uncoated and coated simulations 


\section{COATING WEAR TESTS}

To assess the physical wear characteristics of the three coatings under investigation, a number of tests were conducted using coated samples. The wear rates and failure mechanisms of each coating; A, B \& C, were closely monitored so as to identify the potential improvement in surface durability provided by each respective coating when compared to an uncoated substrate. Coated samples were tested in a rolling contact twin disc rig, each with the same operating conditions, contact load, slide-roll ratio, sample rotational velocity and dry contact. The samples weight loss and surface roughness were measured and optical inspection of the sample surfaces conducted to record the degradation of each test specimen at key milestones throughout the test duration. Wear rate tests were conducted over a range of load cycles (disc revolutions) to provide a more detailed picture of the progressive failure of each coating.

\section{Testing methodology}

A consistent testing procedure was used through the duration of the coating tests to ensure a fair comparison of the wear rate and failure mechanism of each coating. The testing equipment used during this investigation is a bespoke test rig designed and manufactured at Brunel University. Developed specifically to be used for a variety of multi-axis wear tests, minor adaptations were required to make the machine applicable for twin disc testing. The machine is powered by a $1.5 \mathrm{KW}$ three-phase electric motor fitted with a variable output speed controller. The test sample is mounted on a spindle in the sample holder and located directly above the driving disc. The required contact pressure is established by applying a vertical load to the sample holder through a sprung loading arm. Different degrees of rolling-sliding contact can then be induced by turning the sample disc about its loading axis (vertical) relative to the fixed direction of the driving disc. The dimensions of the test sample and driving disc are outlined in figure 7, with further details of the mechanical properties presented in table 2. For the experiments conducted in this investigation, only the test sample discs were coated, and the same uncoated driving disc was used throughout. To reduce wear of the driving disc induced by the high pressure contact conditions, the disc was made from a high carbon steel 
(Orvar H13) which was through-hardened and plasma-nitrided to provide a surface hardness of over 1000HV.

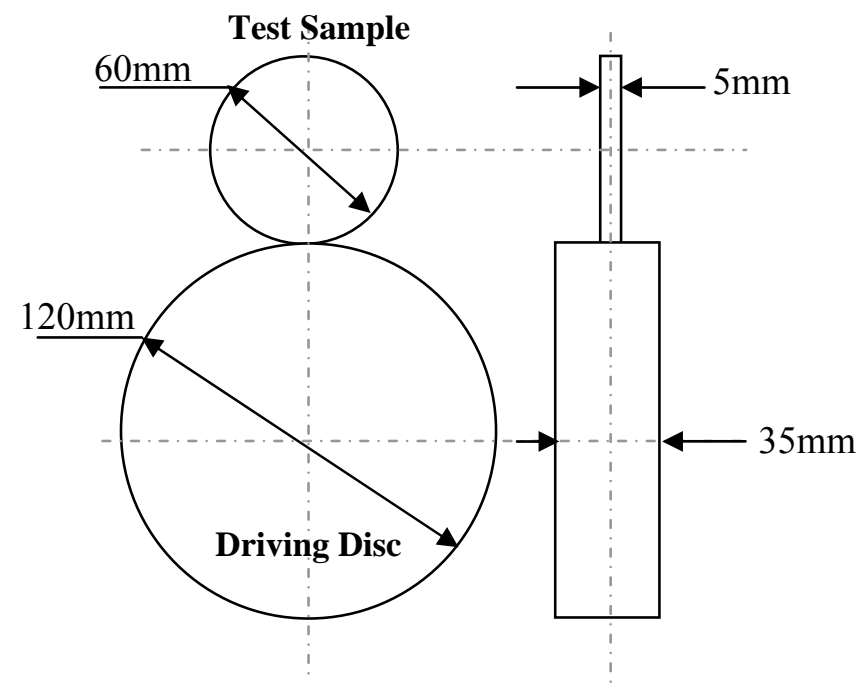

Figure 7 - Dimensions of twin disc test samples and driving disc

Table 2 - Mechanical properties of twin disc components

\begin{tabular}{|c|c|c|c|c|}
\hline Component & Material & $\begin{array}{c}\text { Young modulus } \\
(\mathrm{GPa})\end{array}$ & $\begin{array}{c}\text { Hardness } \\
(\mathrm{HV})\end{array}$ & $\begin{array}{c}\text { Surface roughness } \\
(\mathrm{Ra})\end{array}$ \\
\hline Sample substrate & Vanadis 4 Extra & 206 & 748 & $0.2 *$ \\
\hline Driving disc & $\begin{array}{c}\text { Orvar supreme } \\
\text { H13 }\end{array}$ & 210 & 1000 & 0.05 \\
\hline
\end{tabular}

Each of the samples used in this investigation was tested under the same applied load generating a peak contact pressure of $660 \mathrm{MPa}$ and with a sample rotational speed of 500RPM. Tests were conducted up to a maximum of 145,000 cycles, equivalent to $5 \mathrm{~h}$ continuous running on the rig. To replicate the comparison conducted by the computational simulations, each coating was tested in nonlubricated conditions with a slide-roll ratio of $10 \%$.

\section{Coating Wear Results}

Wear rate tests were conducted over a range of load cycles (disc revolutions) to help provide a more detailed understanding of the progressive failure of each coating. A selection of samples was tested for $30,000,60,000,120,000$ and 145,000 cycles and the mass loss and surface roughness measured 
accordingly. Figure 8 shows the average recorded weight loss for the uncoated and coated test samples over the testing range.

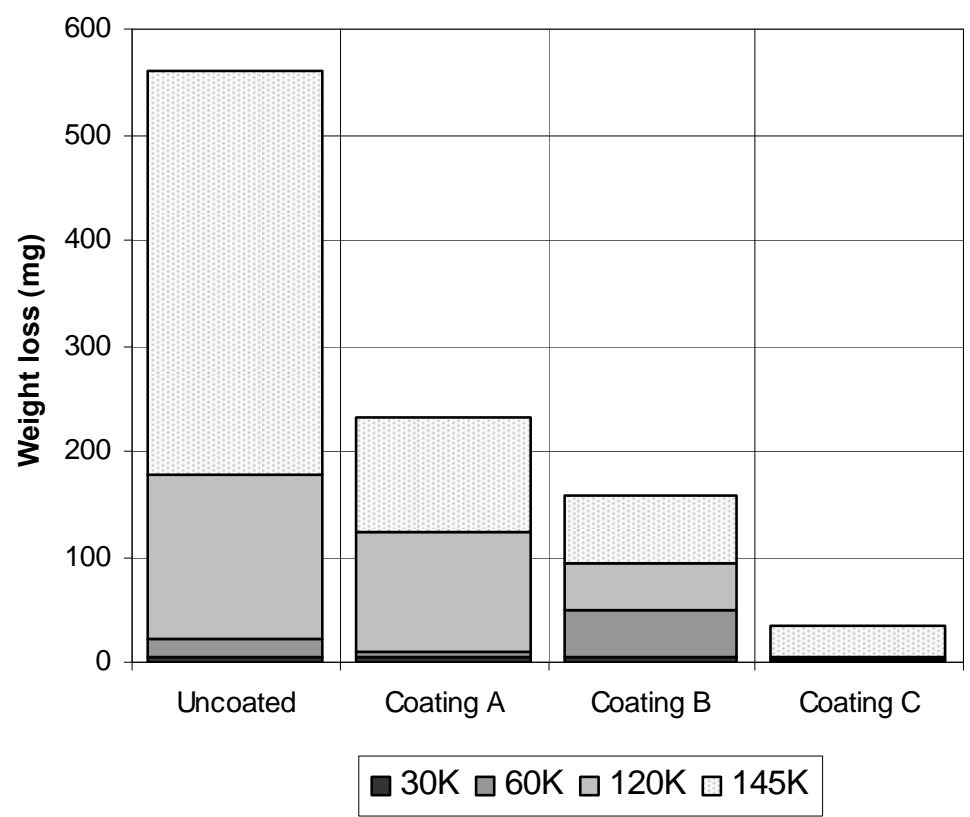

Figure 8 - Cumulative weight loss of test samples over the testing range

It is clear from figure 8 that during the shorter test durations, $30 \mathrm{~K} \& 60 \mathrm{~K}$ cycles, the wear rate of each of the test samples appear reasonably comparable, and very little mass loss has been recorded from the uncoated and coated test samples. This would suggest that each of the samples is wearing at a very similar rate; however, a more detailed optical inspection of the sample surfaces following even the $30 \mathrm{~K}$ cycle test shows some significant signs of damage have already occurred to some of the sample surfaces.

Figure 9 shows a picture of each of the sample surfaces taken with an optical microscope. Surface inspection suggests that the uncoated sample is already showing significant signs of wear, with micropitting and surface damage visible across a significant proportion of the sample surface. Coating A is also showing signs of surface damage after this short run time. Although a significant proportion of the sample surface appears to be in good condition, small areas of micro-pitting damage are visible where the surface coating appears to have been completely removed. Coating B appears to show small pockets of damage occurring in the top layer of the multilayer coating. However, significant proportions of the sample surface appear undamaged at this short test duration. Finally, inspection of 
Coating $\mathrm{C}$ appears to show very little damage with the vast majority of the surface still protected by the coating. Some surface damage is present, however, with small lines of wear visible, in line with the sample rolling direction.
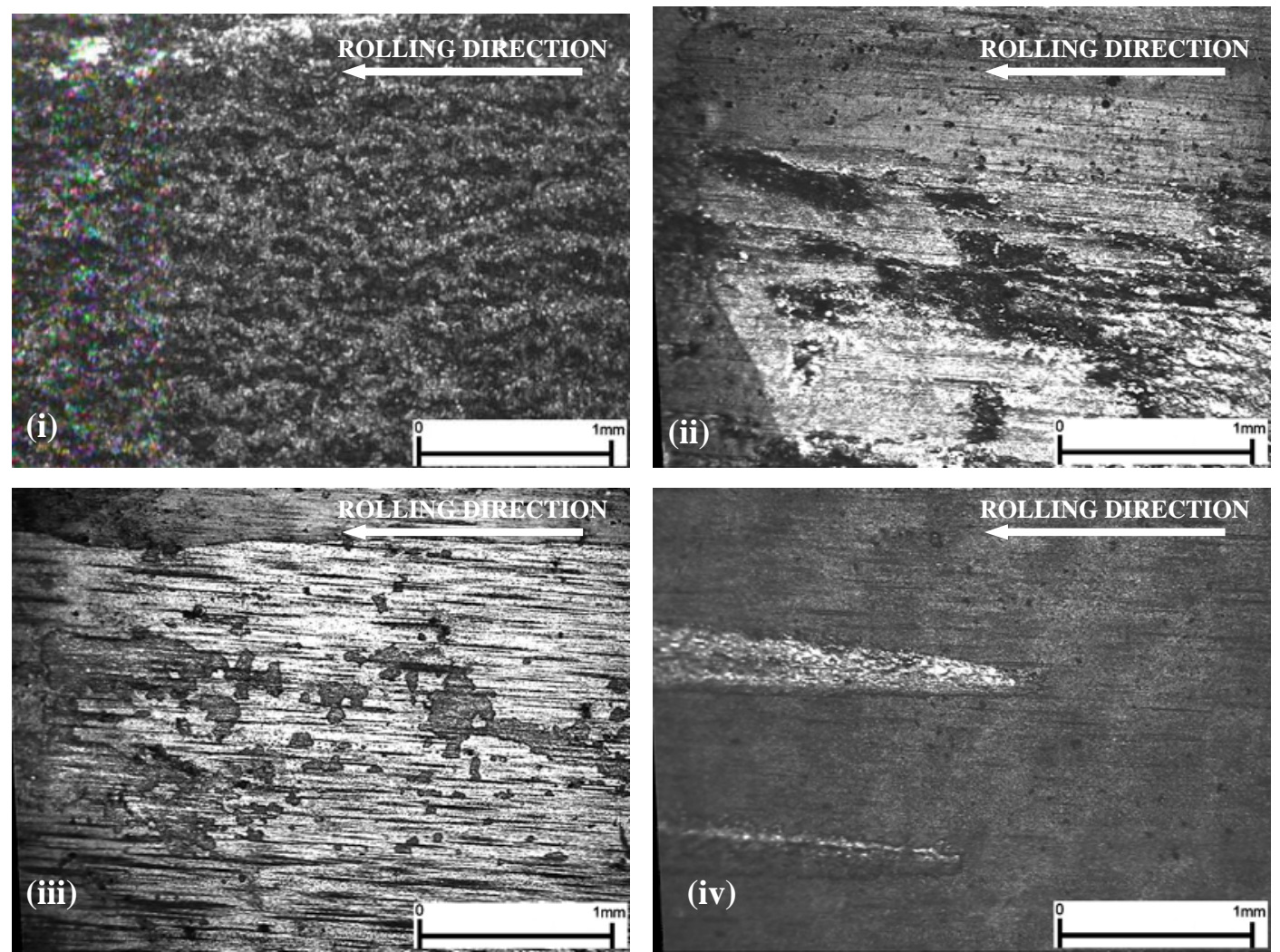

Figure 9 - Optical inspection of sample surface condition after 30,000 cycles, (i) Uncoated, (ii)

Coating A, (iii) Coating B, (iv) Coating C

As the sample test duration increases, the difference in wear between the uncoated and coated samples becomes clearer. Figure10 shows the wear trend of each test sample over the test duration. It is clear that following 120,000 cycles, a significant increase in wear has occurred for all samples except Coating C. A dramatic increase in weight loss has been recorded for both the uncoated and Coating A samples. A more detailed inspection of the surface condition for the uncoated sample shows substantial surface pitting damage has begun to emerge across the vast majority of the sample surface. At the final test duration $(145,000$ cycles), each of the samples tested shows signs of rolling contact fatigue damage. Figure 10 shows that the wear rate of the uncoated sample has continued to increase dramatically as significant surface damage occurs. The average recorded weight loss for Coating A 
has also risen, implying rapid deterioration of the surface condition. Coating B shows a continuation of consistent wear pattern, beginning to increase in magnitude as the test duration extends. Finally, Coating $\mathrm{C}$ is now showing signs of wear as the recorded weight loss increases dramatically. Inspection of the surface of Coating $\mathrm{C}$ at 145,000 cycles showed that all of the coating had been removed leaving the substrate material unprotected. Limited evidence of micro-pitting damage was present on the sample surface.

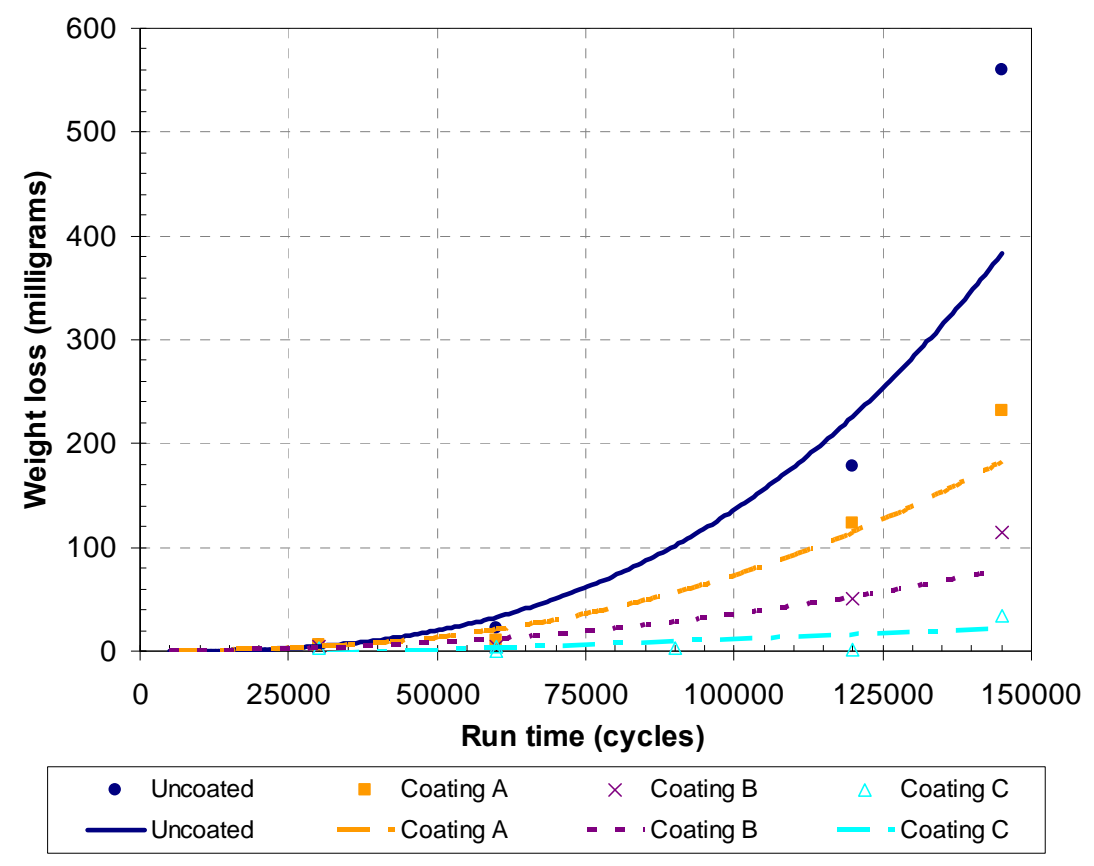

Figure 10 - Wear trend of test samples over duty cycle

\section{CHARACTERISATION OF COATING FAILURE}

To improve the understanding of how each of the coatings failed as a result of the rolling contact fatigue tests, Scanning Electron Microscopy (SEM) techniques were used to closely inspect a number of the samples following testing. A selection of samples was studied and observations of the surface condition recorded. Electron Back-Scatter diffraction and X-ray imaging techniques were also employed to help identify the material elements present and clarify the wear failure mechanisms experienced by each coating. 


\section{Coating A}

Figure 11 is an SEM image of the surface of one of the Coating A test samples following a 30,000 cycle test. Significant damage to the applied coating is clearly visible. Inspection of the sample surface showed that the Titanium-Nitrite coating layer provides such high resistance to wear that it has in fact caused wear on the surface of the driving disc. This was clearly visible on the surface of the driving disc through the creation of a wear track around the disc perimeter. At high magnification, small spot debris particles (from the driving disc) can be identified embedded into the surface of the coating. X-ray inspection of the sample surface identified these particles as small flakes of iron-rich material. This iron debris, created from the wearing effect on the steel driving disc, acts as a 'third body' in the contact, becoming incorporated into the contact patch and causing rapid degradation of the remaining sample coating. In some areas, such a large amount of debris has become embedded in the remaining coating that steel-on-steel contact was effectively established, significantly increasing the interfacial friction between the surfaces and resulting in an increased contact temperature. Discolouration of the TiN layer was observed on some samples as a result of this rise in temperature. However, a significant proportion of the coating does remain intact. Where coating damage is observed, the surface pits that occur were observed to be shallower than the overall thickness of the coating, leaving a thin layer of coating to protect the substrate from wear.
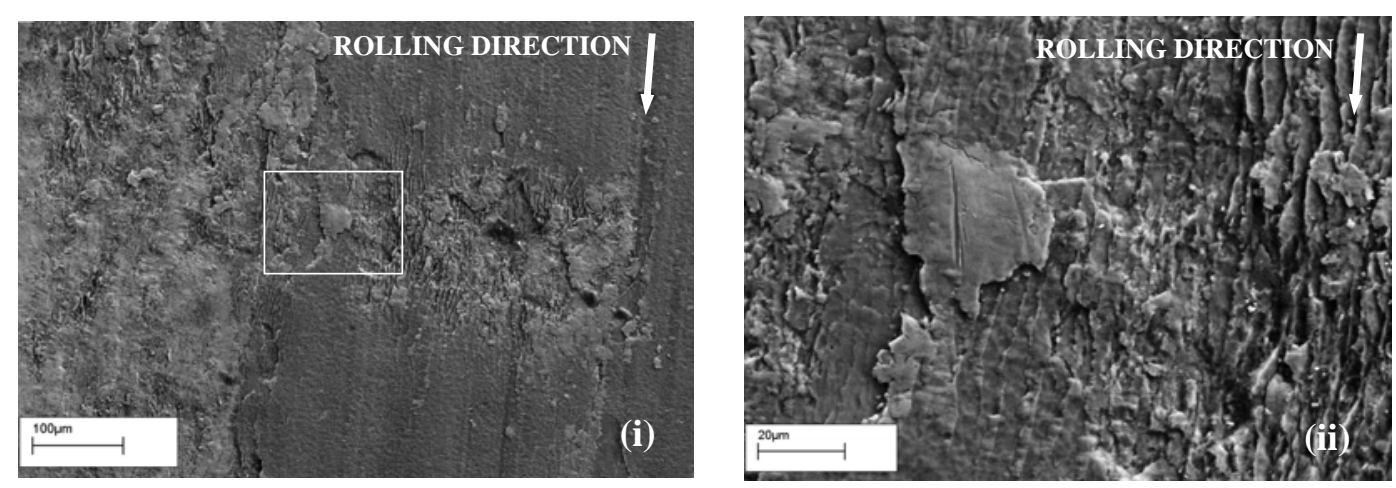

Figure 11 - SEM of coating A test sample after 30,000 cycles (i) x500 magnification (ii) x 2500 magnification 


\section{Coating B}

Due to its composition, coating B has high carbon content. This provides excellent interfacial frictional properties and reduced contact pressures through the removal of damaging surface asperities. However, the Diamond-Like Carbon coating has a material hardness much greater than the substrate, resulting in stress concentrations at the interface. Inspection of the sample surface highlights the presence of numerous small pockets where the DLC coating has been completely removed, exposing the underlying substrate. As the coating continues to wear these pockets are observed to coalesce, forming larger areas of exposed substrate material, as can be seen in figure 12. X-ray inspection of the sample surface indicated the complete removal of all three coating component layers simultaneously, highlighted by the very high presence of Iron in the exposed areas. This would suggest that failure of the coating is occurring as a result of delamination at the thin Chromium interlayer. This interlayer is used as an adhesive aid during the coating application. This type of coating/substrate interface failure is not uncommon for coatings with a hardness value significantly higher than the underlying substrate, as is the case of coating B.
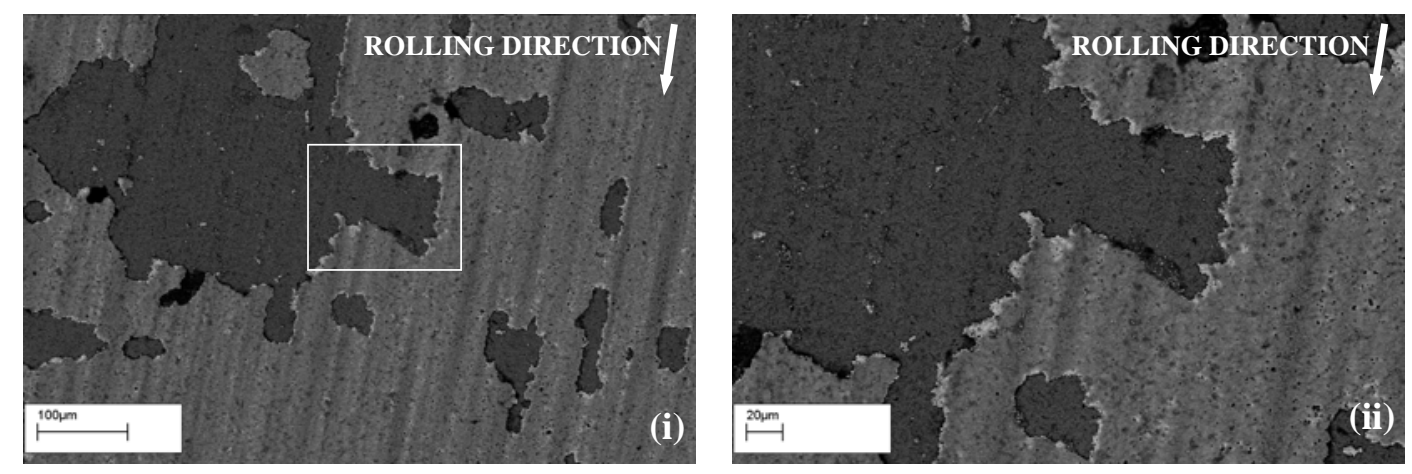

Figure 12 - SEM of coating B test sample after 30,000 cycles (i) x500 magnification (ii) x 2500 magnification

\section{Coating C}

The addition of coating $\mathrm{C}$ was observed to provide excellent protection of the sample surface. Its high carbon composition generates greatly reduced interfacial friction within the contact patch. The softer coating composition also encourages 'running-in' wear. This removes the damaging surface asperities 
that could otherwise cause stress spikes and, instead, spreads the contact load across a larger area, reducing the surface contact stress. Inspection of the sample surface shows very little fatigue damage has occurred, however, some areas of damage are visible (figure 13). Small wear bands can be seen where the WC/C coating appears to have failed. Closer inspection of these wear bands highlights the presence of small curved cracks spreading from the tips of the wear bands. This suggests the coating is breaking into small flakes at the tips of the wear bands, causing the bands to grow in length. X-ray inspection of one of these wear bands indicates a high presence of Chromium, suggesting that although the Tungsten Carbide/Carbon layer has warned away, the underlying Chromium interlayer appears to remain intact. This would imply that the high contact stresses are causing delamination of the WC/C layer from the Cr layer. The emergence of the wear bands coincides with the locations of the grinding marks visible from the underlying substrate surface. This suggests that failure of the $\mathrm{WC} / \mathrm{C}$ layer may be promoted by the stress concentrations present around the edges of the grinding scores.
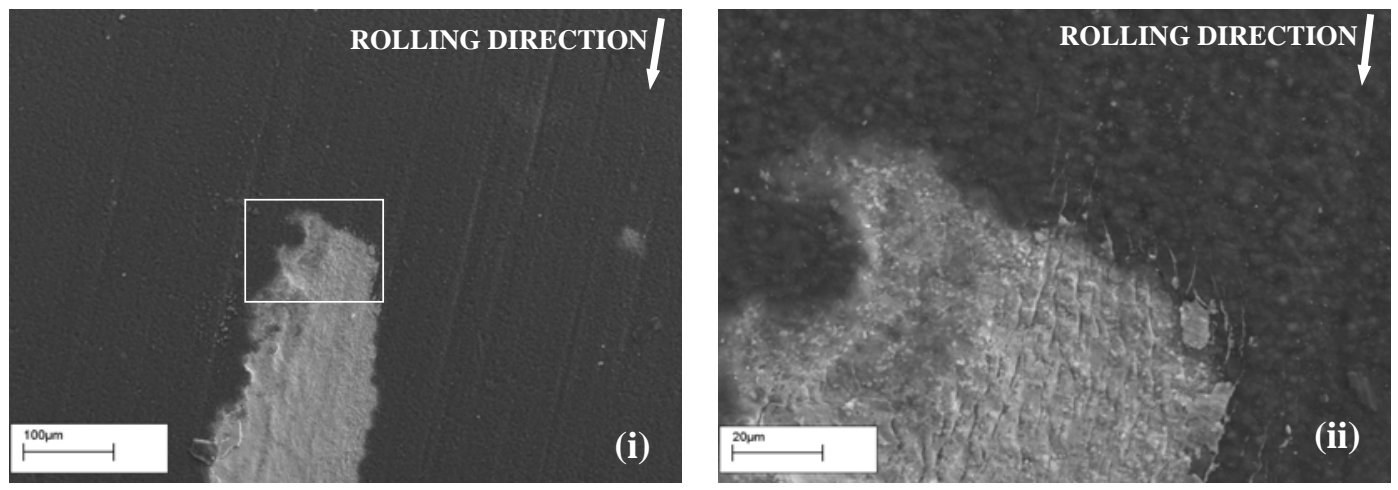

Figure 13 - SEM of coating $C$ test sample after 30,000 cycles (i) x500 magnification (ii) x 2500

\section{magnification}

Throughout the tests conducted, coating $\mathrm{C}$ was found to offer excellent resistance to rolling contact fatigue, greatly improving the usable life of the coated component and providing excellent surface durability. Inspection of the driving disc even showed evidence of transfer of a thin carbon film from the test samples, resulting in the very low friction contact between the two surfaces, figure 14. This 
phenomenon has been frequently observed in tests of high carbon content coatings against uncoated counterparts [12].

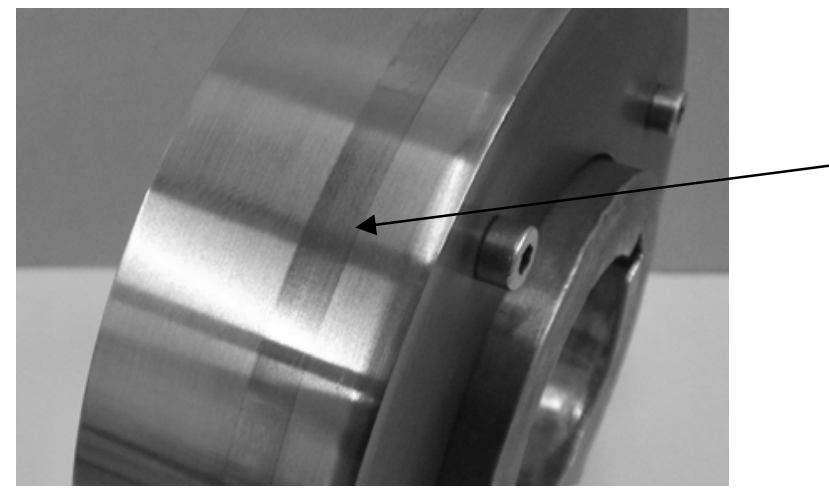

\section{Carbon transfer from Coating $C$ test sample}

Figure 14 - Transfer of carbon film from Coating $C$ to driving disc (30,000 cycles)

All the coatings have been shown to provide a significant improvement in wear resistance when compared to the uncoated substrate. The high hardness of coating A is very wear resistant, protecting the surface of the substrate. However, under the conditions tested here, this was found to induce wear in the driving disc creating wear debris that became incorporated into the coating, eventually promoting failure of both the driver and sample surfaces. The low friction and high hardness properties from its Diamond-Like Carbon composition make coating B very resistant to wear in rolling contact fatigue conditions. Observation of the coating failure mechanics does, however, suggest that the high hardness of the coatings creates stress concentrations at the coating/substrate interface, encouraging delamination of the coating layers and resulting in the failure of the protective surface. It is clear from the direct comparison of each of the three coatings that coating $\mathrm{C}$ provides the most significant improvement in surface durability under the rolling-contact fatigue conditions tested. Optical inspection of the sample surfaces has shown that the coating remains intact for a substantial proportion of the run times tested. The much softer composition of coating $\mathrm{C}$ reduces the coating/substrate interface stress concentration, improving adhesion of the coating to the substrate. For coating C, wear appears to occur as a result of delamination between the Tungsten Carbide/Carbon layer and Chromium interlayer, possibly induced by stress concentrations occurring around the residual grinding marks on the underlying substrate. 


\section{CONCLUSION}

In the first part of this paper, a multilayer contact FE model was developed which was shown to provide significant improvements in the understanding of how the addition of complex multilayer coatings responds to mixed rolling-sliding contact in dry conditions. The method of modelling each layer as a separate part provides the capability to rapidly adjust layer geometry and material properties in the model and the potential to simulate an essentially limitless number of surface layers. Consequently, a direct comparison can be made between a variety of different coating configurations quickly and easily with the alteration of key layer properties such as thickness, hardness, elastic modulus, surface friction and roughness. The result is a versatile tool offering a rapid and quantitative comparison of a number of coating configurations under a specific set of loading conditions. Furthermore, through the use of advanced finite element software, a number of critical output variables such as stresses, strains, plastic deformation, contact pressure, contact area and contact temperature can be closely monitored and compared throughout the entire model, or just in the regions of interest. This provides the means to compare the effects of the stresses and strains experienced by the underlying substrate material, providing a wealth of information that can be used to carefully design components to maximise the benefits available from different surface coatings.

In the second part of this paper, results were presented from the physical testing of the same three advanced coatings used in the computational modelling. Each of the coatings was tested over a range of test durations to establish a picture of their progressive wear rates in dry contact conditions. From the twin disc experiments conducted in this study, it has become clear that each of the coatings tested offered significant improvements in surface durability when compared to the uncoated test samples. This is particularly true for the high Carbon-content coatings, Coating B and Coating C. Both coatings showed excellent resistance to rolling contact fatigue failures with noticeably reduced surface damage and recorded sample weight loss. From the rolling contact fatigue experiments conducted in this study, Coating C provided the most significant increase in surface durability with a 16-fold reduction in measured weight loss compared to the uncoated substrate over the 145,000 cycle test duration investigated. 


\section{ACKNOWLEDGMENTS}

The work described in this paper was supported by the EPSRC. The authors would also like to thank the following companies for their assistance with various parts of this research: Oerlikon Balzers Coatings Ltd, Uddeholm \& Abaqus Inc.

\section{REFERENCES}

[1] Texas Automotive Manufacturing Industry Report, "http://www.governor.state.tx.us", February 2007.

[2] Crain Communications, Automotive News, March 15, 2004.

[3] Taylor, C.M. (1998), 'Automobile engine tribology — design considerations for efficiency and durability', Wear, 221, 1-8.

[4] Komvopoulos, K. (1989), 'Elastic-plastic finite element analysis of indented layered media', Journal of Tribology, 111, 430-439.

[5] Mao, K., Sun, Y. \& Bell, T. (1994), 'Contact mechanics of engineering: State of the art', Surface Engineering, 10, 297-306.

[6] Holmberg, K., Laukkanen, A., Ronkainen, H., Wallin, K., Varjus, S. \& Koskinen, J. (2006), 'Tribological contact analysis of a rigid ball sliding on a hard coated surface Part 1: Modelling stresses and strains', Surface \& Coatings Technology, 200, 3793-3809.

[7] Djabella, H. \& Arnell, R. (1993), 'Finite-element analysis of contact stresses in elastic doublelayer systems under normal load', Thin Film Solids, 226, 65-73.

[8] Dong, H., Bell, T. \& Mynott, A. (1999), 'Surface engineering of titanium alloys for the motorsport industry', Sports Engineering, 2, 213-219.

[9] Bloyce, A. \& Michler, T. (2002), 'PVD coatings for gears', Heat Treatments of Metals, 29, 33-38.

[10] Walton, D. \& Goodwin, A.J. (1998), 'The wear of unlubricated metallic spur gears', Wear, 222, 103-113.

[11] Abaqus Analysis User's Manual version 6.4 (2002), Hibbitt, Karlsson \& Sorensen Inc, USA. 
[12] Amaro, R.I., Martins, R.C., Seabra, J.O., Renevier, N.M. \& Teer, D.G. (2005), 'Molybdenum Disulphate/Titanium low friction coating for gear applications', Tribology International, 39, 423-434. 